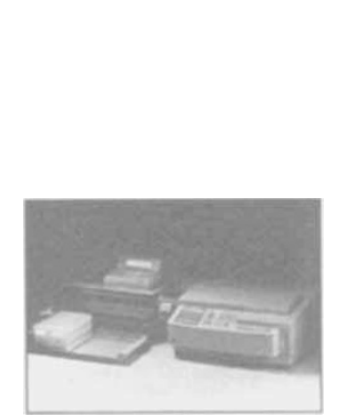

Autosampler

ATI Unicam (Cambridge, U.K.) has launched a new autosampler system, based on the Gilson XL, for use with its UV Series of UV/Visible spectrometers. Used with the UV Series local control keypad or with Windows software, results processing can be performed while the autosampling system is in operation.

Write in 970 on Reader Service Card.

\section{High-intensity lamps}

Varian (San Fernando, CA) introduces UltrAA Lamps, a range of highintensity, boosted-discharge, hollowspectroscopy. The lamps use a secondary discharge to achieve a higher light emission output resulting in reduced baseline noise levels that improve detection limits.

Write in 973 on Reader Service Card. cathode lamps for atomic absorption

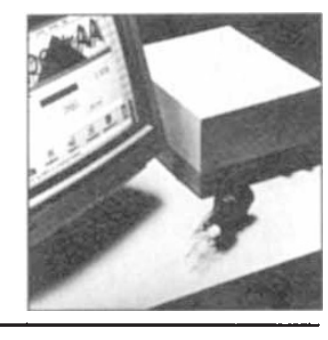

\section{Spectrometer}

Leeman Labs (Lowell, MA) offers ICP direct reading sequential spectrometers featuring Echelle grating. This measuring system can be up to three times faster than conventional systems. Analytical reliability is maximized with the direct reading sequential system.

Write in 975 on Reader Service Card.

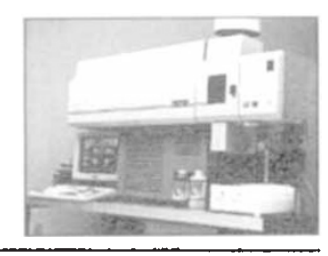

\section{Kinetics measurements}

Hi-Tech Scientific (Salisbury, U.K.), introduces the SF-61 DX-2, a computer-controlled, stopped-flow system for rapid kinetics measurements. It features an automated sample handling unit and KinetAsyst, a Windows-based control package. The DX2 has a range of detector options suitable for both absorbance and fluorescence measurements.

Write in 972 on Reader Service Card.

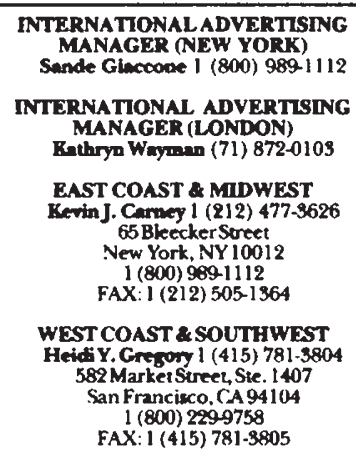

\section{Rapid imaging}

With Astromed's (Cambridge, U.K.) parallel clock accelerator, the 4200 series of cooled CCD cameras, equipped with the EEV CCD37, can acquire up to one million spectra per second. If light from the emission slit of a spectrograph illuminates 8 rows of the $C C D$, the system acquires 127 spectra at intervals of 8 microseconds. Write in 971 on Reader Service Card.

NORTHERN GERMANY
Andre Kichler (40) $\$ 800-137$
Prof-Brix-Weg 8. D-22767 Germany
FAX: (40) $3800-166$
SCANDINAVIA

$$
\begin{aligned}
& \text { dra Hewandt (8) 6440005 } \\
& \text { Andrew Kamir \& Associates } \\
& \text { Finnbodavagen 1. 13131 NACKA Sweden } \\
& \text { FAX: (8) } 6-42.31 .50 \\
& \text { FRANCE/HOLLANDAELGIUM } \\
& \text { Patricis Hummel (1) 43-87-42-17 } \\
& 5 \text { Rue Joseph Sansboeuf } \\
& 75008 \text { Paris France } \\
& \text { SWITZERLAND } \\
& \text { Verene LoewenthalHugd (41) } 5461.9090 \\
& \text { Internationale Mediaberatung. Postfach } \\
& \begin{array}{l}
\text { CH-8267 Berlingen } \\
\text { FAX: (41) } 5461 \$ 200
\end{array}
\end{aligned}
$$

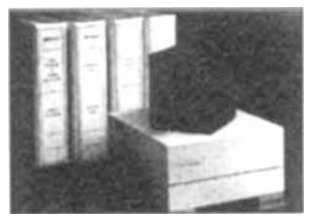

JAPAN

Philif Hamin (3) 267.8751 Shin-Mitsuke Building (4F) 36 Ichigaya Tamachi FAX: (3) 2678746

$$
\text { AUSTRALIA }
$$

Ampe Murrey (2) 299-5677 World Media Network Pty, Level 89 York Sireet, Sydney. NSW 2000 FAX: (2) 2996178 ISRAEL Heiko Sieter, Intermedii (3) 328.7815 76 King George St. PO BUX 11820 Tel Aviv, Israel 611116
FAX: (3) 299259 